\title{
Reactividad y propiedades mecánicas de escoria de alto horno activada por álcalis
}

\author{
J.I. ESCALANTE-GARCIA, J. MÉNDEZ-NONELL, A. GOROKHOVSKY, \\ P. E. FRAIRE-LUNA, H. MANCHA-MOLINAR, G. MENDOZA-SUAREZ \\ Centro de Investigación y de Estudios Avanzados del IPN, Unidad Saltillo, Saltillo Coahuila, México
}

\begin{abstract}
Se realizaron estudios de caracterización de reactividad y propiedades mecánicas en pastas de escoria de alto horno activada por 5 sistemas alcalinos como $\mathrm{NaOH}$, vidrio soluble y combinaciones de $\mathrm{Na}_{2} \mathrm{CO}_{3^{\prime}}, \mathrm{Na}_{2} \mathrm{SO}_{4}$ y $\mathrm{Ca}(\mathrm{OH})_{2}$ en concentraciones de $5 \%$. Se prepararon cubos de $5 \mathrm{~cm}$ por lado y se curaron durante 120 días a $20^{2}$ y $60^{\circ} \mathrm{C}$ bajo agua. Los valores mas altos de resistencia mecánica fueron registrados por el sistema de escoria activada con vidrio soluble, seguido por una de las combinaciones de 3 reactivos químicos, mientras que el desarrollo de propiedades mecánicas fue más pobre con la activación por $\mathrm{NaOH}$. El aumento en la temperatura de curado favoreció la resistencia a la compresión de escoria con vidrio soluble, superando a la del cemento Portland a todos los tiempos de curado; para los otros sistemas el incremento de la temperatura de curado resultó perjudicial. En contraste con las observaciones de resistencia mecánica, la reactividad observada por mediciones de agua no evaporable y disolución química selectiva indicaron que la escoria es mas reactiva en presencia de $\mathrm{NaOH}$. Las imágenes por Microscopía electrónica de barrido utilizando electrones retrodispersados confirmaron las diferentes reactividades y las propiedades mecánicas observadas.
\end{abstract}

Palabras clave:Escoria, activación alcalina, microestructuras, productos de hidratación, reactividad

\section{Reactivity and mechanical properties of alkali activated blast furnace slag}

Investigations were carried out to characterize the reactivity and mechanical properties in blast furnace slag pastes activated by 5 alkali systems such as $\mathrm{NaOH}$, waterglass and combinations of $\mathrm{Na}_{2} \mathrm{CO}_{3}, \mathrm{Na}_{2} \mathrm{SO}_{4}$ y $\mathrm{Ca}(\mathrm{OH})_{2}$ in concentrations of $5 \%$. Cubes of $5 \mathrm{~cm}$ were prepared and cured under water for up to 120 days at 20 and $60^{\circ} \mathrm{C}$. The highest compressive strengths were noted for the waterglass activation, followed by one of the combination of 3 compounds, while $\mathrm{NaOH}$ activation resulted in the lowest strengths. Increased temperatures favored the strength for the waterglass activation, outperforming those of the Portland cement paste used as control at all times, whereas it had a negative effect for all other activations. The reactivity of the systems, as obtained by measurements of non evaporable water and selective chemical dissolution, indicated that the slag is more reactive under the $\mathrm{NaOH}$ activation. Scanning electron microscopy by means of backscattered electrons confirmed the different reactivities and the mechanical properties observed

Key words: Blast furnace slag, alkali activation, microstructures, hydration products, reactivity

\section{INTRODUCCIÓN}

Entre los diversos problemas asociados al crecimiento de la población y a la generación de bienestar está el consumo de recursos naturales y la generación de contaminación. Una de las formas de reducir el impacto al medio ambiente es por medio de la reutilización y reciclado de desechos y subproductos industriales. En materiales de construcción, la escoria de alto horno activada por álcalis representa una posibilidad de producir materiales de características superiores a las de aquellos hechos con cemento portland (1), en cuanto a propiedades mecánicas y durabilidad $(2,3)$. Adicionalmente, como dicha escoria es un subproducto de la producción de arrabio (4), solo se requiere de enfriamiento (5) y tamaño de partícula -molienda- (6) adecuados para poder usarse; por lo que las emisiones ambientales perjudiciales asociadas son mínimas comparadas con la producción del cemento Portland.

La escoria de alto horno tiene propiedades hidráulicas y puede reaccionar con el agua pero de forma muy lenta. Para potenciar dichas propiedades, una de las formas de activación es la química (7). En esta se produce la rotura de la red vítrea de la escoria por iones $\mathrm{OH}^{-}$, disolviendo los componentes de la escoria con la posterior precipitación de productos de reacción (8) que poseen carácter cementicio y son insolubles en agua. Entre los activantes más comúnmente aplicados están el vidrio soluble (silicato de sodio), $\mathrm{NaOH}, \mathrm{Na}_{2} \mathrm{CO}_{3^{\prime}}, \mathrm{Na}_{2} \mathrm{SO}_{4^{\prime}}$ $\mathrm{Ca}(\mathrm{OH})_{2}$ y combinaciones de estos.(9-14) Generalmente se dice que la activación por $\mathrm{NaOH}$ resulta en las propiedades mecánicas más bajas y aquella con vidrio soluble en propiedades superiores a las del cemento Portland. La activación con $\mathrm{Na}_{2} \mathrm{CO}_{3}, \mathrm{Na}_{2} \mathrm{SO}_{4}, \mathrm{Ca}(\mathrm{OH})_{2}$ resultó en propiedades similares a las obtenidas con $\mathrm{NaOH}$, sin embargo no se especificó la composición del activante.(15)

Los productos de reacción resultantes en función del activante, así como de las propiedades mecánicas y microestructurales, pocas veces se estudian de manera asociada. Por otro lado, existen pocos trabajos acerca del efecto de la temperatura sobre la reactividad de la escoria activada y los productos de reacción formados para escorias activadas con varios agentes alcalinos. Este trabajo presenta resultados del estudio de 
los efectos de la temperatura y del tipo de activante sobre las características y propiedades mencionadas de escorias de alto horno activadas.

\section{PARTE EXPERIMENTAL}

\subsection{Materiales}

Se utilizó una escoria granulada de Alto Horno proveniente de la planta AHMSA (Monclova, Coahuila México), suministrada por Cementos Apasco (planta Ramos Arizpe) en donde fue acondicionada, en un molino de bolas piloto, para obtener un área superficial de $450 \mathrm{~m}^{2} / \mathrm{kg}$. La composición química de los principales componentes (\% peso) fue: $34.5 \mathrm{SiO}_{2}$, $33.6 \mathrm{CaO}, 10.2 \mathrm{Al}_{2} \mathrm{O}_{3^{\prime}}, 10.2 \mathrm{MgO}, 4.7 \mathrm{Fe}_{2} \mathrm{O}_{3^{\prime}}, 4.2 \mathrm{SO}_{3}$. El halo en la región de 20 a $60^{\circ} 2 \theta$, observado en el patrón de difracción presentado en la Figura 1, indica que la escoria es predominantemente amorfa; se notó la presencia de algunas fases cristalinas menores como Akermanita $\left(\mathrm{Ca}_{2} \mathrm{MgSi}_{2} \mathrm{O}_{7}\right)$, Gehlenita $\left(\mathrm{Ca}_{2} \mathrm{Al}_{2} \mathrm{SiO}_{7}\right)$, Merwinita $\left(\mathrm{Ca}_{3} \mathrm{MgSi}_{2} \mathrm{O}_{8}\right)$ y Hematita $\left(a-\mathrm{Fe}_{2} \mathrm{O}_{3}\right)$. Como referencia se empleó un cemento portland ordinario cuya composición en términos de los principales óxidos fue: $20.3 \mathrm{SiO}_{2}, 61.3 \mathrm{CaO}, 4.7 \mathrm{Al}_{2} \mathrm{O}_{3}, 1.7 \mathrm{MgO}, 2.9 \mathrm{Fe}_{2} \mathrm{O}_{3}, 3.5 \mathrm{SO}_{3}$.

Los agentes activantes empleados fueron vidrio soluble,

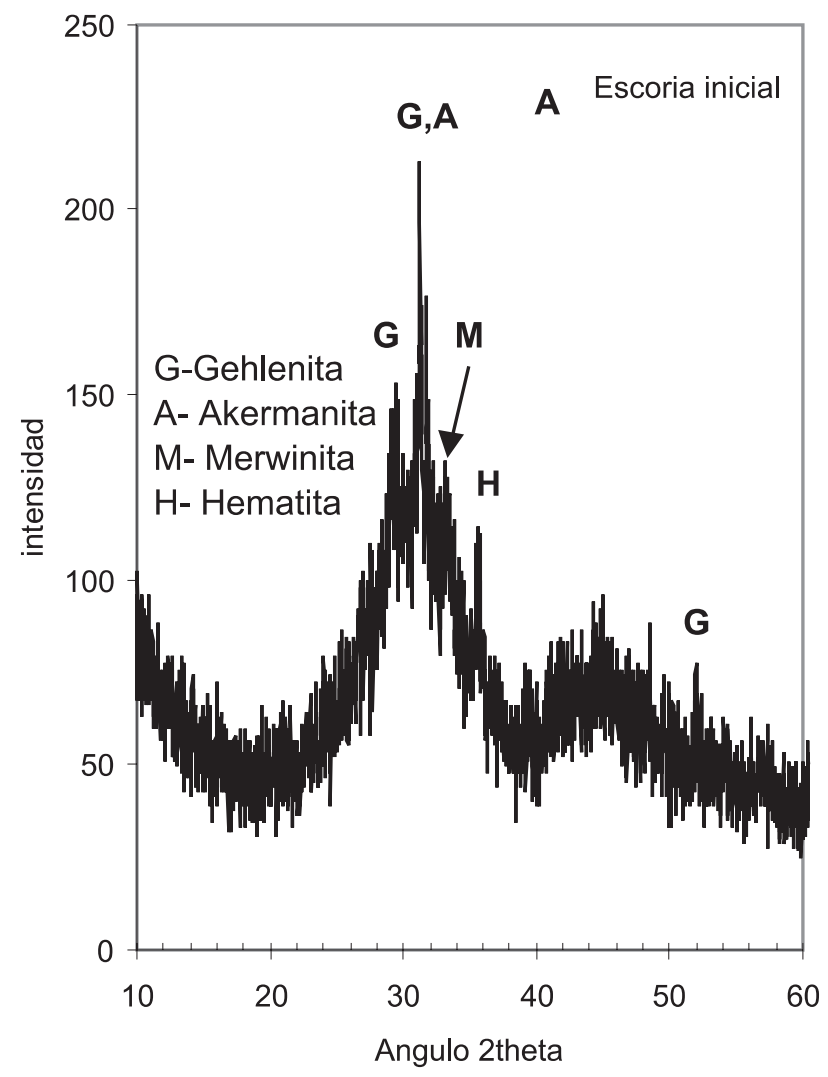

Figura 1 Patrón de difracción de rayos X de la escoria sin reaccionar

$\mathrm{NaOH}$ y tres combinaciones de $\mathrm{Na}_{2} \mathrm{CO}_{3}, \mathrm{Na}_{2} \mathrm{SO}_{4}$ y $\mathrm{Ca}(\mathrm{OH})_{2}$ con composiciones porcentuales pare este último (así referidas en el resto del documento) 50-25-25, 25-50-25 y 25-50-50 en el mismo orden.

\subsection{Hidratación y curado de las muestras}

Antes de la preparación de las pastas de escorias activadas, se prepararon las disoluciones de los agentes activantes, empleando la concentración requerida de $5 \%$ con respecto al peso de la escoria (el vidrio soluble se agregó en $5 \%$ de $\mathrm{Na}_{2} \mathrm{O}$ ). Adicionalmente se prepararon muestras con $10 \%$ de $\mathrm{NaOH}$. La cantidad de agua utilizada fue de $0.4 \mathrm{~kg}$ agua $/ \mathrm{kg}$ escoria, los $\mathrm{pH}$ medidos de las soluciones se presentan en la tabla 1

TABLA 1 pH DE LAS SOLUCIONES EMPLEADAS COMO ACTIVANTES.

\begin{tabular}{|l|l|l|l|l|l|l|}
\hline & \multicolumn{2}{|c|}{$\mathrm{NaOH}$} & $\begin{array}{l}\text { Vidrio } \\
\text { soluble }\end{array}$ & \multicolumn{2}{c|}{$\mathrm{Na}_{2} \mathrm{CO}_{3}-\mathrm{Na}_{2} \mathrm{SO}_{4}-\mathrm{Ca}(\mathrm{OH})_{2}$} \\
\hline & $5 \%$ & $10 \%$ & $5 \%$ & $50-25-25$ & $25-50-25$ & $25-25-50$ \\
\hline $\mathrm{pH}$ & 13.21 & 13.41 & 12.49 & 13.19 & 13.13 & 13.12 \\
\hline
\end{tabular}

La mezcla de la escoria con las disoluciones se llevó a cabo en un agitador de paleta durante 4 minutos y posteriormente se vaciaron las pastas en moldes de Nylacero®, resistentes a los álcalis. Las mezclas se vibraron durante 1 minuto para procurar la eliminación de burbujas atrapadas. Los moldes, ya llenos, se recubrieron con un plástico y franelas húmedas para evitar pérdidas de agua por evaporación. Los moldes llenos se dejaron durante 24 horas en cámaras isotérmicas a $20 \pm 2$ y $60 \pm 3{ }^{\circ} \mathrm{C}$ para fraguado. Dichas temperaturas se seleccionaron para estudiar el efecto de esta en la velocidad y mecanismos de las reacciones, asi como en las propiedades mecánicas. Posteriormente, se desmoldaron los cubos y se sumergieron en agua a las mismas temperaturas dentro de las mencionadas cámaras; iniciando así el periodo de curado. Adicionalmente, como referencia de las propiedades mecánicas se prepararon pastas de cemento Portland ordinario con relación de agua/cemento de 0.4 .

\subsection{Caracterización}

Para cada uno de los períodos de curado considerados de 28, 90 y 120 días, se midió la resistencia a la compresión de 4 cubos por muestra utilizando una prensa hidráulica, haciendo el promedio de los datos. Con el objetivo de detener las reacciones de hidratación, de los fragmentos de los cubos se separó una alícuota de aproximadamente $100 \mathrm{~g}$ y se trituró manualmente en mortero con pistilo a un tamaño aproximado de $1 \mathrm{~mm}$. Las muestras se secaron a $105^{\circ} \mathrm{C}$ en una estufa de vacío durante 12 horas, iniciando el calentamiento al arrancar la operación de la bomba de vacío. Posteriormente, las muestras secas se pasaron por molienda manual hasta pasar una malla de $150 \mathrm{~mm}$.

El grado de avance de las reacciones se calculó a partir de la cantidad de agua no evaporable (ANE) (16). El procedimiento implica la calcinación de una muestra, de aproximadamente un $1.000 \mathrm{~g}$, a $950^{\circ} \mathrm{C}$ durante 30 minutos y se utilizó la siguiente fórmula $\mathrm{ANE}=100 \times\left(\mathrm{PesO}_{105^{\circ} \mathrm{C}}-\mathrm{PesO}_{950^{\circ} \mathrm{C}}\right) / \mathrm{PesO}_{950^{\circ} \mathrm{C}}$. Para evaluar la cantidad de escoria reaccionada se empleó el método reportado por Luke y Glasser (17),utilizado por Escalante y Cols. (18). El método se basa en el uso de ácido etilen diamino tetraacético e hidróxido de sodio, para disolver selectivamente los productos de hidratación dejando la escoria sin reaccionar. Luke y Glasser(17) evaluaron varios métodos de este tipo e indicaron que este era el mas confiable. El método se utilizó inicialmente para mezclas de cemento portland con escoria de alto horno, por lo que la fórmula de cálculo de la cantidad de escoria reaccionada se modificó a la siguiente forma: 

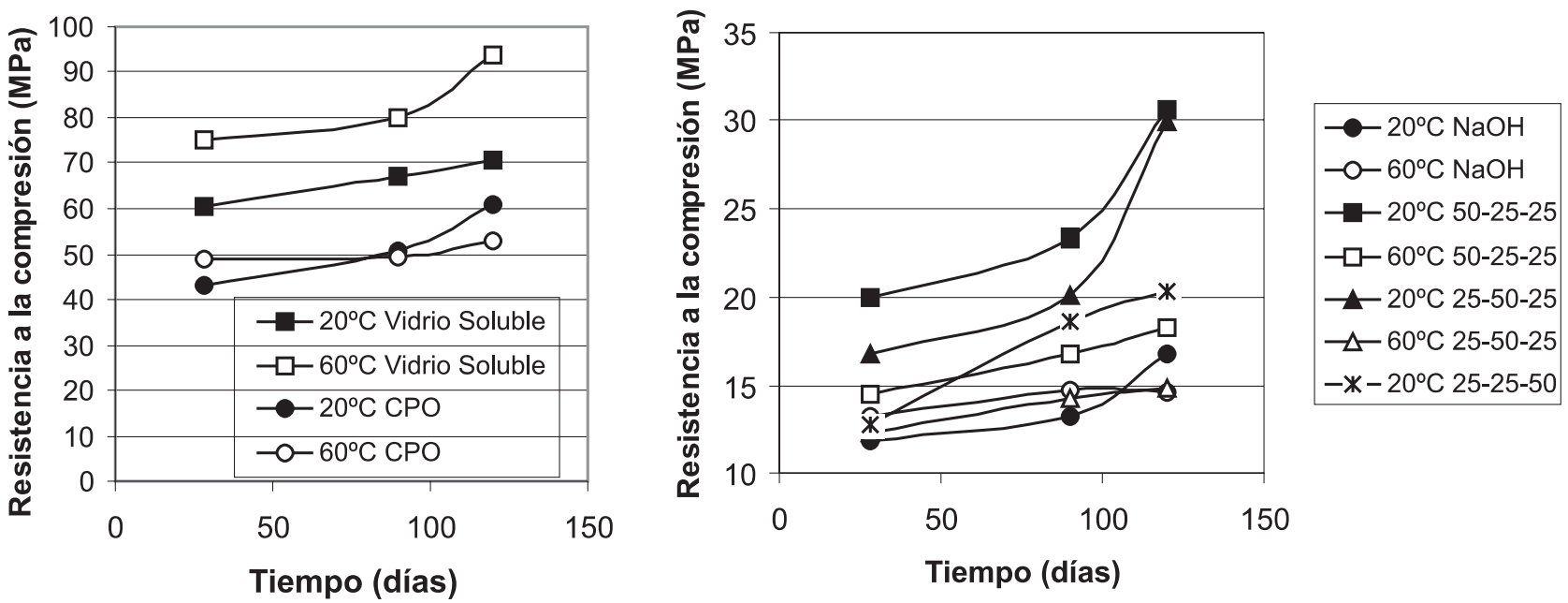

Figura 2 Resistencia a la compresión vs tiempo para la escoria activada con los diversos agentes activantes a 20 y $60^{\circ} \mathrm{C}$. CPO-cemento portland ordinario, los activantes indicados con números como 50-25-25 indican la composición de este en $\mathrm{Na}_{2} \mathrm{CO}_{3}-\mathrm{Na}_{2} \mathrm{SO}_{4}-\mathrm{Ca}(\mathrm{OH})_{2}$, $\mathrm{respectivamente}$

$$
\% \operatorname{Re}=\frac{P i-P f *(1+\varepsilon)}{\mathrm{Pi}} * 100
$$

Donde: $\% \mathrm{Re}=$ porcentaje de reacción de la escoria, $\mathrm{Pi}=$ peso inicial de la escoria hidratada $(0.2500 \mathrm{~g}) ; \mathrm{Pf}=$ peso final de la escoria después de la disolución, e=factor de corrección 0.08

Por medio de pruebas de la aplicación del método de disolución química selectiva sobre la escoria sin reaccionar, se encontró que el método disuelve un porcentaje de ésta. Después de 7 repeticiones se determinó que se disolvía un 8\%, dicho porcentaje se adaptó en la fórmula anterior como factor de corrección. No se realizaron correcciones por fases de hidratación no disueltas por el método (como la hidrotalcita) que pueden llevar a errores de en la aplicación del método (17).

Para la caracterización por microscopía electrónica (Jeol 6300), se emplearon trozos de los fragmentos de ensayos mecánicos y se montaron en resina epóxica. Las muestras se desbastaron y pulieron con pasta de diamante de hasta $1 / 4 \mu \mathrm{m}$ usando un lubricante libre de agua; finalmente se recubrieron de carbón para hacerlas conductoras en el microscopio.

\section{RESULTADOS Y DISCUSIÓN}

\subsection{Resistencia a la compresión}

La Figura 2 presenta los resultados de resistencia a la compresión de las pastas durante 28, 90 y 120 días, con los 5 agentes activantes a 20 y $60^{\circ} \mathrm{C}$ (para $25-25-50$ solo a $20^{\circ} \mathrm{C}$ en vista de que de la combinación de tres álcalis dio las propiedades mecánicas más bajas después de 90 días). Puede notarse que, entre las escorias activadas, los valores de resistencia a la compresión mas altos resultaron para el vidrio soluble (superiores a las del cemento Portland), seguidos por la activación a $20^{\circ} \mathrm{C}$ con 50-25-25. Los resultados más pobres fueron para la activación con $\mathrm{NaOH}$.

El incremento de la temperatura resultó muy favorable para la activación con vidrio soluble, con ganancias entre 24$28 \%$ para los tiempos indicados. En contraste, el incremento en la temperatura resultó en la reducción de la resistencia a la compresión para el resto de los sistemas - incluyendo al cemento Portland. Los efectos de la temperatura sobre el desarrollo de resistencia mecánica en cementos portland han sido discutidos en algunas publicaciones. $(19,20)$ A altas temperaturas la resistencia mecánica es mayor en las etapas de curado iniciales dado que, como es de esperarse, las reacciones de hidratación ocurren a mayor velocidad; sin embargo, la rápida formación de productos de hidratación impide que estos alcancen a difundir y depositarse en forma homogénea (en contraste con la hidratación a bajas temperaturas) dejando poros en la microestructura. Dicha porosidad hace que en largo plazo, la resistencia mecánica de cemento portland curado a temperaturas más bajas sea mayor. Para el $\mathrm{NaOH}$ el incremento de la temperatura resultó ligeramente favorable durante 28 y 90 días solamente, decayendo después de 120 días.

\subsection{Reactividad de la escoria}

La Figura 3 muestra los resultados de agua no evaporable (ANE), empleados como una medida de la reactividad del sistema. Puede notarse que la cantidad de ANE se incrementa con el tiempo, lo que indica que las reacciones progresan generando productos de hidratación. En contraste con los resultados de resistencia mecánica descritos anteriormente, los valores de ANE para la activación con $\mathrm{NaOH}$ estuvieron ligeramente por encima de los del vidrio soluble a los 28 y 90 días, lo cual se invirtió a los 120 días para $60^{\circ} \mathrm{C}$. El efecto del incremento de la temperatura es similar al observado para las propiedades mecánicas: favorable para la activación con vidrio soluble pero desfavorable para el resto de los sistemas

La figura 4 presenta los resultados del porcentaje de escoria reaccionada por activación con los diversos agentes. Similar a los datos de ANE, los resultados indican que la cantidad de escoria sin reaccionar disminuye con el tiempo para dar lugar a productos cementivos. En este caso puede observarse que los valores mas altos de reactividad son para la escoria activada con $\mathrm{NaOH}$, seguidos por la combinación 5025-25 y en último lugar por el vidrio soluble. 

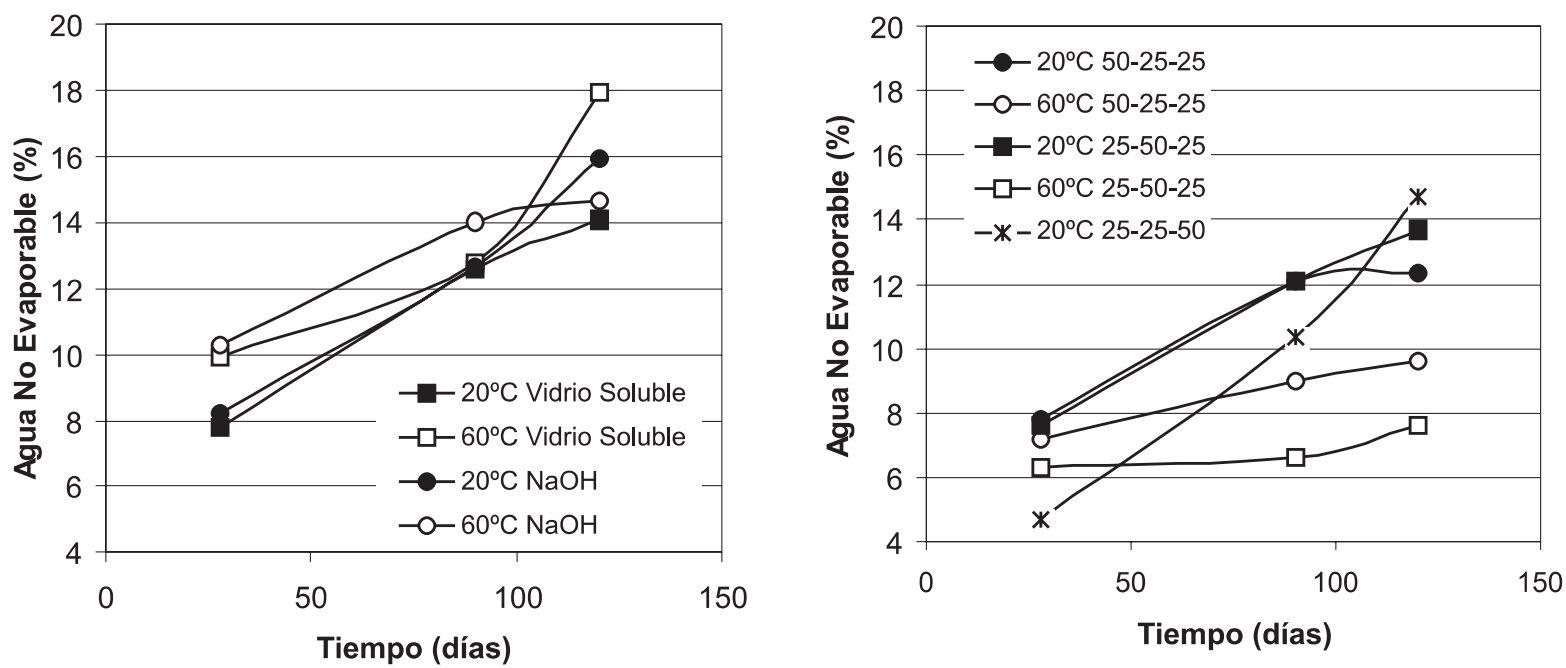

Figura 3 Agua no evaporable vs tiempo para la escoria activada con los diversos agentes activantes a 20 y $60^{\circ} \mathrm{C}$. Los activantes indicados con números como 50-25-25 indican la composición de este en $\mathrm{Na}_{2} \mathrm{CO}_{3}-\mathrm{Na}_{2} \mathrm{SO}_{4}-\mathrm{Ca}(\mathrm{OH})_{2}$, respectivamente

En la figura 3 puede notarse que los valores de ANE correspondientes a la activación con vidrio soluble no son los más bajos, como sucede en la figura 4 al respecto de la cantidad de escoria reaccionada. Esto puede explicarse dado que el ANE es una medida de la cantidad de agua químicamente enlazada, que incluye el agua presente en los productos formados, independientemente de la activación de la escoria, que por parte del vidrio soluble puede ser un gel de sílice, dicho gel adicionalmente favorece microestructuras mas compactas y por ende propiedades mecánicas mayores (9). En contraste, la disolución química selectiva aplicada hace seguimiento solo de la cantidad de escoria sin reaccionar y puede ser considerada como una medida mas cierta sobre la reactividad, solo de la escoria.

\subsection{Fases de hidratación formadas}

La figura 5 presenta los patrones de difracción de rayos $X$ de la escoria activada con los diversos agentes activantes y curada durante 90 días a 20 y $60^{\circ} \mathrm{C}$. Para ambas temperaturas se aprecia la presencia de un pico difuso alrededor de los $30^{\circ} 2 \theta$ que se sobrepone al halo amorfo de la escoria sin reaccionar, dicho pico es atribuible al gel C-S-H, como se ha puesto de manifiesto en otros trabajos $(8,21-23)$; dicho gel es el principal responsable de las propiedades cementicias de la escoria y que también es producido por la hidratación del cemento Portland. Se puede observar que a $20^{\circ} \mathrm{C}$ los picos del C-S-H son menos intensos en el caso del sistema activado con vidrio soluble, infiriendo una menor reactividad de la escoria en este sistema, en concordancia con los resultados de reactividad de la escoria(Fig. 4).

$\mathrm{Al}$ incrementar la temperatura de curado a $60^{\circ} \mathrm{C}$ se notó que los tipos de productos de hidratación formados fueron básicamente similares al curado a $20^{\circ} \mathrm{C}$. Sin embargo, el pico del gel C-S-H apareció mas marcado y agudo. Esto puede sugerir, por un lado, que la cantidad del gel C-S-H aumenta al elevar la temperatura o bien que es la cristalinidad del C$\mathrm{S}-\mathrm{H}$ la que aumenta, lo cual es difícil diferenciar con esta información y además existen pocas referencias en la literatura al respecto.

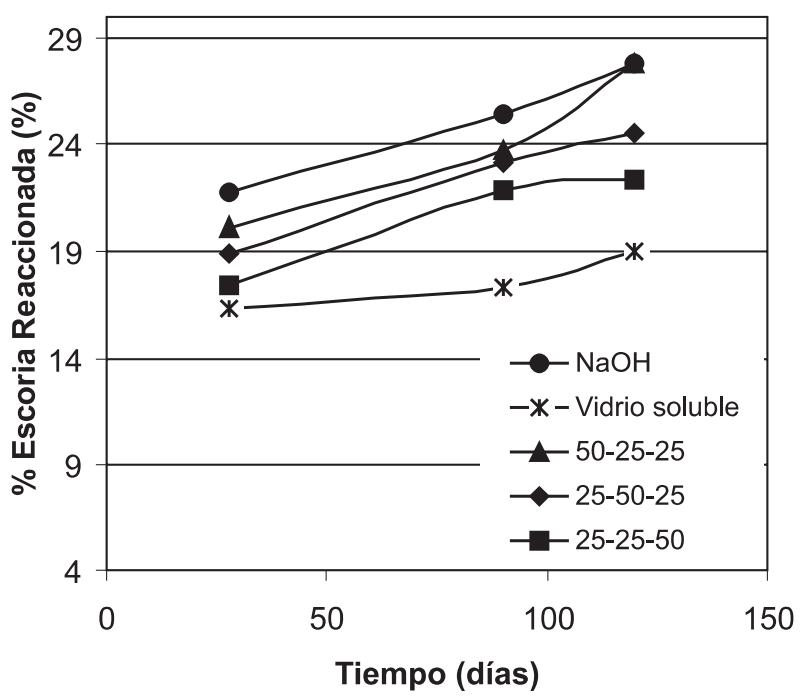

Figura 4 Porcentaje de escoria reaccionada vs tiempo para la escoria activada con los diversos agentes activantes a $20^{\circ} \mathrm{C}$. Los activantes indicados con números como 50-25-25 indican la composición de este en $\mathrm{Na}_{2} \mathrm{CO}_{3}-\mathrm{Na}_{2} \mathrm{SO}_{4}-\mathrm{Ca}(\mathrm{OH})_{2}$, respectivamente

Otra de las fases observadas fue una fase tipo hidrotalcita $\left(\mathrm{Mg}_{6} \mathrm{Al}_{2} \mathrm{CO}_{3}(\mathrm{OH})_{16} \cdot 4 \mathrm{H}_{2} \mathrm{O}\right)$ que también ha sido reportada como producto de la hidratación de las escorias activadas $(8,24)$ dados los contenidos de $\mathrm{Mg}$ y Al.

\subsection{Microestructuras}

Para el análisis microestructural se utilizó la microscopía electrónica de barrido empleando imágenes por electrones retrodispersados. Este tipo de imágenes permite distinguir las fases presentes por medio del contraste químico, en el que las fases de mayor número atómico promedio se ven más claras. 

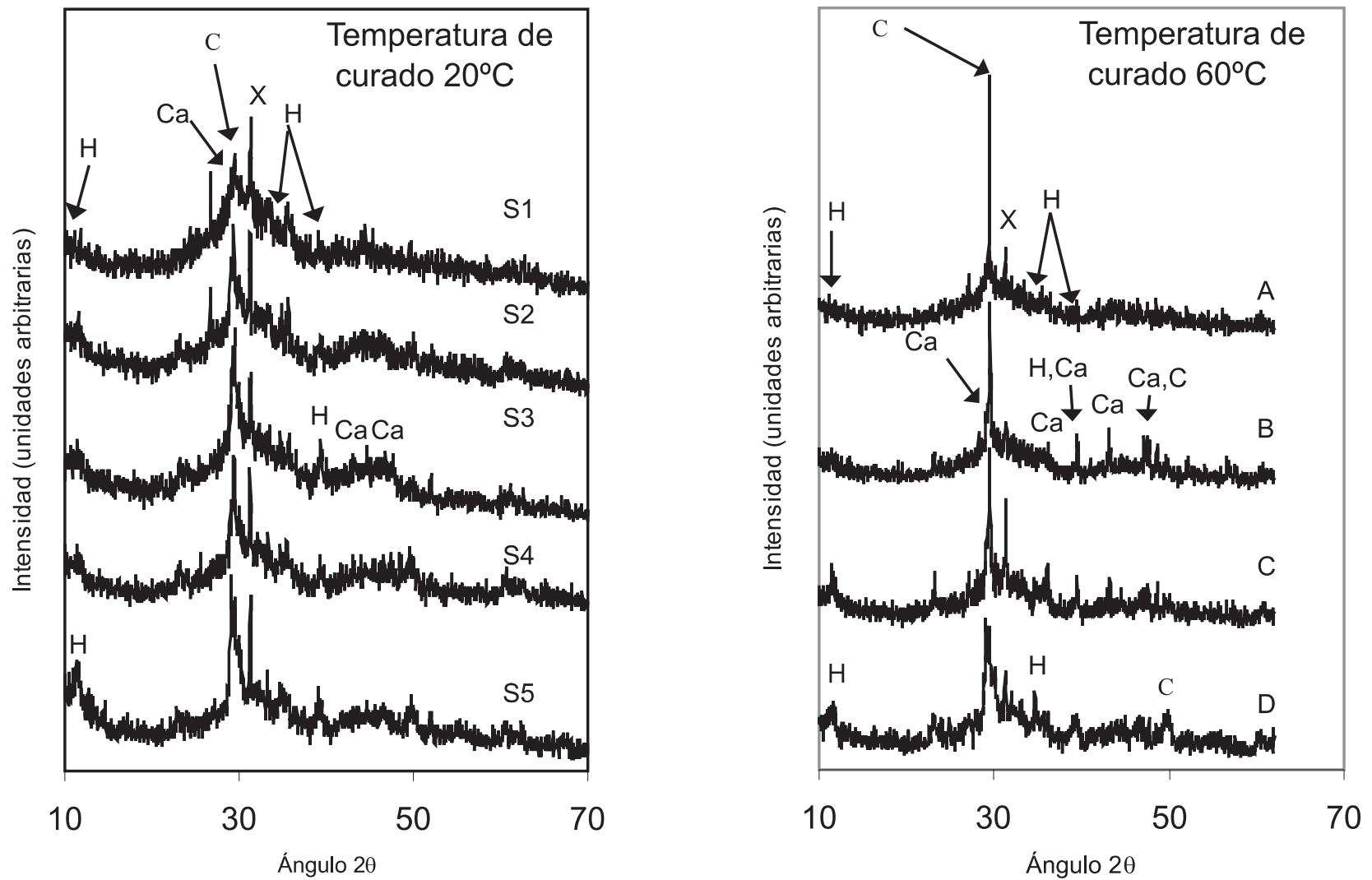

Figura 5 Patrones de difracción de rayos $\mathrm{X}$ para la escoria curada con los diversos agentes activantes por 90 días a 20 y $60^{\circ} \mathrm{C}$. Se indican los picos principales. Nomenclatura $\mathrm{C}(\mathrm{C}-\mathrm{S}-\mathrm{H}), \mathrm{H}$ (Hidrotalcita), $\mathrm{Ca}\left(\mathrm{CaCO}_{3}\right), \mathrm{X}$ (escoria sin reaccionar). Nomenclatura agentes activantes $\mathrm{S} 1$ (vidrio soluble), S2 (25-25-50), S3 (25-50-25), S4 (50-25-25), S5 ( NaOH)

Así, es posible distinguir los granos de escoria, por su tono más claro (y por la morfología angular irregular), de los productos de hidratación que aparecen en un tono gris más obscuro, mientras que los poros se ven de color negro. Para este estudio se seleccionaron solamente muestras activadas con vidrio soluble, $50-25-25$ y NaOH durante 90 días a 20 y $60^{\circ} \mathrm{C}$. Los resultados se presentan en la figura 6.

Respecto a la influencia del tipo de activante puede verse que las estructuras más compactas y continuas fueron las obtenidas por el sistema activado con vidrio soluble (fotos A y B); las grietas observadas se produjeron durante secado y también fue común observar la aparición de grietas durante la observación en el vacío del microscopio electrónico. Algunos "poros" aparentes en la escoria con vidrio soluble (foto A) son granos de escoria arrancados durante el pulido, lo que puede deducirse por su morfología. En contraste, las escorias activadas con 50-25-25 (foto $\mathrm{C}$ ) y $\mathrm{NaOH}$ (Foto E) tienen elevada porosidad, en concordancia con los resultados de la resistencia a la compresión (Fig. 2).

El incremento de la temperatura de curado para la escoria con vidrio soluble resultó en una matriz aparentemente mas compacta que a $20^{\circ} \mathrm{C}$. Una comparación cuidadosa de las fotos A y B indica que a $60^{\circ} \mathrm{C}$ existen mas granos de escoria parcial- mente hidratados, que pueden identificarse por el "anillo" más obscuro de productos de hidratación alrededor del centro blanco anhídro. Algunos granos de escoria aparecieron totalmente hidratados a $60^{\circ} \mathrm{C}$, lo que no fue observado a $20^{\circ} \mathrm{C}$; esto esta en acuerdo con las observaciones de agua no evaporable al respecto del incremento en la cantidad de productos de reacción al incrementar la temperatura. Dichas fotografías parecen indicar que el mecanismo predominante de reacción de la escoria a $20^{\circ} \mathrm{C}$ es por ataque alcalino y disolución de su red vítrea, con la consecuente precipitación de los productos de hidratación en los espacios inicialmente ocupados por el agua. Pocos granos reaccionaron por el mecanismo en estado sólido a $20^{\circ} \mathrm{C}$, en el cual los reactantes difunden a través de la capa de productos de hidratación ya formada, consumiendo los granos de escoria hacia el interior (formando los anillos previamente mencionados) y precipitando los productos de reacción dentro de las fronteras originales de los granos.

Para la activación con 50-25-25, el incremento de temperatura produjo un aparente aumento en la porosidad, dado que a $60^{\circ} \mathrm{C}$ fue común observar poros en la microestructura (notar poros grandes del lado derecho centro y arriba). Esto puede explicar la reducción de las propiedades mecánicas a 90 días, discutida anteriormente. Para este sistema es posible notar 

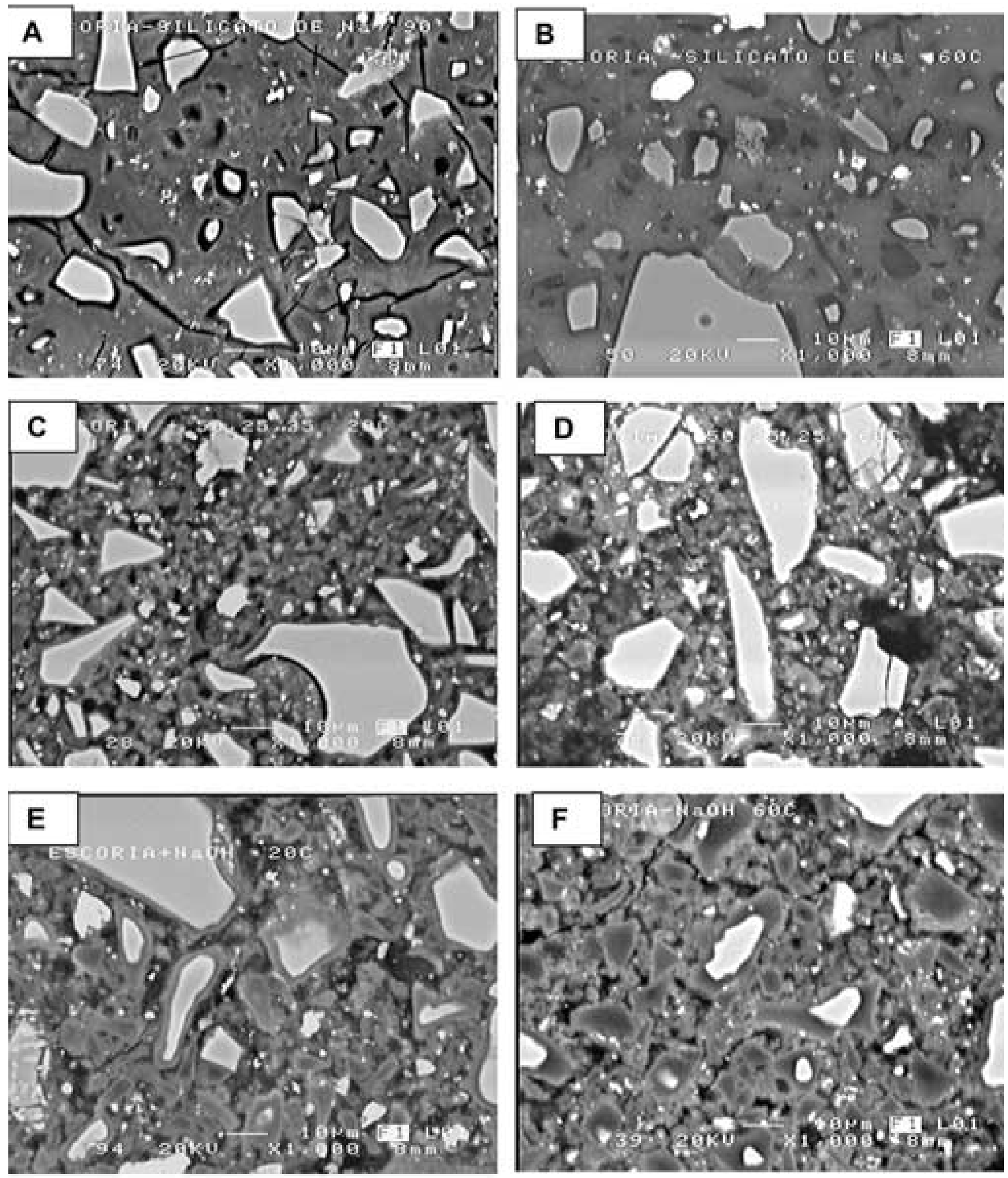

Figura 6 Micrografías obtenidas por microscopía electrónica de barrido para muestras de escoria curadas durante 90 días y activadas con (A) Vidrio Soluble a $20^{\circ} \mathrm{C}$, (B) Vidrio Soluble a $60^{\circ} \mathrm{C}$, (C) $50 \% \mathrm{Na}_{2} \mathrm{CO}_{3}-25 \% \mathrm{Na}_{2} \mathrm{SO}_{4}-25 \% \mathrm{Ca}(\mathrm{OH})_{2}$ a $20^{\circ} \mathrm{C}$, (D) $50 \% \mathrm{Na}_{2} \mathrm{CO}_{3}-25 \% \mathrm{Na}_{2} \mathrm{SO}_{4}-25 \% \mathrm{Ca}(\mathrm{OH})_{2}$ a $60^{\circ} \mathrm{C}$, (E) $\mathrm{NaOH}$ a $20^{\circ} \mathrm{C}$ y (F) $\mathrm{NaOH}$ a $60^{\circ} \mathrm{C}$ 
que el mecanismo de reacción predominante fue por disolución y precipitación (descrito anteriormente), la mayoría de los granos de menor tamaño presentan reacción en estado sólido.

Para el caso de la activación con $\mathrm{NaOH}$ no se observó un claro incremento en la porosidad. A diferencia de los otros dos sistemas, se observó que muchos de los granos de la escoria se encuentran parcialmente reaccionados por el mecanismo de reacción en estado sólido (descrito antes). Esto puede notarse al apreciar la gran cantidad de granos de escoria que tienen un centro anhídro (blanco) y un anillo de productos de reacción dentro de la frontera original de los granos. Al aumentar la temperatura, se puede observar que existe una mayor cantidad de granos totalmente hidratados, principalmente los granos de menor tamaño.

\subsection{Correlación de Resultados.}

Como se ha indicado, las propiedades mecánicas mejores y más pobres fueron obtenidos para la activación con vidrio soluble y $\mathrm{NaOH}$, respectivamente. De acuerdo con las observaciones microestructurales, las muestras con mayor porosidad fueron aquellas activadas con $\mathrm{NaOH}$, en comparación con las activadas con vidrio soluble. Sin embargo, los resultados de ANE (figura 3) no estuvieron en total acuerdo con esto, los valores de ANE con $\mathrm{NaOH}$ y vidrio soluble fueron similares. Por otro lado, para la cantidad de escoria activada (figura 4) los mayores niveles de reacción se alcanzaron por activación con $\mathrm{NaOH}$ y los menores con vidrio soluble. Este panorama de resultados puede sugerir que aunque la escoria es mas fuertemente atacada por el $\mathrm{NaOH}$ que por el vidrio soluble, en el primer caso la predominancia del mecanismo de reacción en estado sólido puede limitar la saturación de los espacios inicialmente ocupados por agua con productos de reacción, dejando una alta porosidad remanente. Por otro lado, es posible que los productos formados con $\mathrm{NaOH}$ sean de menor resistencia mecánica intrínseca que los formados con vidrio soluble. Adicionalmente, aunque el vidrio soluble aparentemente ataca en menor grado a la escoria, éste contribuye con productos que saturan la porosidad para dar matrices densas y compactas con excelentes propiedades mecánicas. La reducción de las propiedades mecánicas y de ANE con 50-25-25 al incrementar la temperatura puede haber resultado de un incremento en la solubilidad y lixiviación del activante en las pastas (curadas bajo agua) a tiempos cortos de curado (bajo nivel de reacción y alta porosidad), la falta de activante pudo así haber limitado la formación de productos cementicios y por ende dejando poros en la microestructura.

\section{CONCLUSIONES}

Las mejores propiedades mecánicas fueron para la escoria activada con vidrio soluble (sobrepasando las del cemento Portland), seguidas por la activación con $50 \% \quad \mathrm{Na}_{2} \mathrm{CO}_{3}-25 \%$ $\mathrm{Na}_{2} \mathrm{SO}_{4}-25 \% \mathrm{Ca}(\mathrm{OH})_{2}$ mientras que las mas bajas fueron para activación con $\mathrm{NaOH}$.

El incremento en la temperatura resultó en un aumento en la resistencia a la compresión para la activación con vidrio soluble, mientras que para los otros sistemas resultó lo contrario en el largo plazo. El mismo patrón se observó con las mediciones de agua no evaporable, que es equivalente a la cantidad de agua químicamente enlazada.
Las fases formadas en todos los sistemas fueron gel C-S-H y una fase tipo hidrotalcita $\left(\mathrm{Mg}_{6} \mathrm{Al}_{2} \mathrm{CO}_{3}(\mathrm{OH})_{16} \cdot 4 \mathrm{H}_{2} \mathrm{O}\right)$.

Las microestructuras observadas para la activación con vidrio soluble fueron más densas y compactas mientras que la activación con $\mathrm{NaOH}$ y $50 \% \quad \mathrm{Na}_{2} \mathrm{CO}_{3}-25 \%-\mathrm{Na}_{2} \mathrm{SO}_{4}-$ $25 \% \mathrm{Ca}(\mathrm{OH})_{2}$ resultó en microestructuras altamente porosas a las dos temperaturas de curado, en acuerdo con la mayor resistencia a la compresión de la escoria activada con vidrio soluble.

Se observó que para la activación con $\mathrm{NaOH}$ las reacciones se llevaron intensamente por medio del mecanismo de reacción en estado sólido, en el cual los granos de escoria se consumen hacia adentro y los productos de reacción se depositan al interior de la frontera de los granos individuales, observándose una intensa reacción de la escoria. El mecanismo por disolución-precipitación con la precipitación de los productos de reacción en los espacios inicialmente ocupados por el agua parece operar en menor grado. Para la activación con vidrio soluble y $50 \% \mathrm{Na}_{2} \mathrm{CO}_{3}-25 \% \mathrm{Na}_{2} \mathrm{SO}_{4}-25 \% \mathrm{Ca}(\mathrm{OH})_{2}$ se observó que el mecanismo predominante fue el de disoluciónprecipitación

\section{AGRADECIMIENTOS}

Se agradece al Consejo Nacional de Ciencia y Tecnología el apoyo por medio del proyecto J28273U, al Ing. Serafín Martínez de Cementos Apasco por el apoyo otorgado y al Ing. M Aguilar por el apoyo en la operación del microscopio electrónico.

\section{BIBLIOGRAFIA}

1. D.M Roy, "alkali activated cements, Opportunities and challenges", Cem Concr Res 29 (1999) 249-254

2. C. Shi, "Strength, pore structure and permeability of alkali activated slag mortars", Cem Concr Res 26 (1996) 1789-1799

3. S.D Wang, K L Scrivener, "Factors affecting the strength of alkali activated slag", Cem Concr Res 24 (1994) 1033-1043

4. M Regourd, "Cement made from blast furnace slag", en "LEA's chemistry of cement and Concrete, Ed P C Hewlett, Arnold London 1997

5. C R Wilding, G Mchugh, The hydration of blast furnace cements", Atomic Energy Research Establishment, Harwell, 1986, AERE Report R 12297

6. C. Frigione, "Manufacture and characteristics of portland blast furnace slag cements", in Blended Cements, ASTM STP 897, Ed G Frohnsdorff, American Society for Testing and Materials, Philadelphia 1986 pp 15-28

7. M Regourd, "Slag and Slag cement", in Concrete Technology and Design, Ed. R.N Swamy, Vol 3 Cement Replacement Materials, pp 73-98, Surrey University Press, 1986

8. S D Wang, K L Scrivener, "Hydration products of alkali activated slag cement", Cem Concr Res, 25 (1995) 561-571

9. S D Wang, K L Scrivener, "Factors affecting the strength of alkali activated slag", Cem Concr Res, 24 (1994) 1033-1043

10. C Shi, "Strength, pore structure and permeability of alkali activated slag mortars", Cem Concr Res 26 (1996) 1789-1799

11. P S Parameswaran, A K Chatterjee, "Alkali acivation if indian blast furnace slags", Proc $8^{\text {th }}$ international congress on the chemistry of cement, Vol 4, pp 86-91, Rio de Janeiro Brazil, 1986

12. A Fernandez-Jimenez, J G Palomo, F Puertas, "Alkali activated slag mortars Mechanical strength behaviour", Cem Concr Res, 29 (1999) 1313-1321

13. F Collins, J G Sanjayan, "early age strength and workability of slag pastes activated by $\mathrm{NaOH}$ and $\mathrm{Na}_{2} \mathrm{CO}_{3}{ }^{\prime \prime}$, Cem Concr Res, 28 (1998) 655-664

14. T Bakharev, J G. Sanjayan, Y B Cheng, Alkali activation of australian slag cements", Cem Concr Res 29 (1999) 113-120

15. P.S Parameswaran y A K Chatterjee, Alkali activation of indian blast furnace slags, Proceedings $8^{\text {th }}$ International Congress on the Chemistry of Cement, Vol. 4 pp 86-91, Rio de Janeiro Brazil 1996

16. H.F. W Taylor, Cement Chemistry, 2ª Edición Thomas Telford Londres 1997 
17. K luke, F. P Glasser, Selective dissolution of hydrated blast furnace slag cements, Cem Concr Res 17 (1987) 273-282

18. J I Escalante, L Y Gómez, K K Johal, G Mendoza, H Mancha, J Méndez, Reactivity of blast-furnace slag in portland cements blends hydrated under different conditions", Cem Concr Res 31 (2001) 1403-1409

19. K O Verbeck, R.H. Helmuth, Structures and physical properties of cement paste, Proceedings of the $5^{\text {th }}$ International congress on the Chemistry of Cement, pp 1-32, Tokyo, Japan 1968

20. J I Escalante-Garcia , J H Sharp, Effect of temperature on the hydration of the main clinker phases in portland cement: Part I, neat cements, Cem. Concr. Res. 28 (1998) 1245-1257

21. P J Shilling, A Roy, H V Eaton, P G Malone, W N Brabston, Microstructure, strength and reaction products of ground granulated blast-furnace slag activated by highly concentrated $\mathrm{NaOH}$ Solution, J Mater Res, Vol 9, No 1, Jan 1994, pp188-197

22. A.R. Brough, M Holloway, J Skyes, A Atkinson, Sodium silicate based alkali activated slag mortars Part II, The retarding effect of additions of sodium chloride and malic acid, Cem Concr Res 30 (2000) 1375-1379

23. A. Roy, P J shilling, H C Eaton, P G Malone, W N Brabston, L D Wakeley, Activation of ground blast-furnace slag by alkali -metal and alkaline-earth hydroxides, J Am Ceram Soc 75 [12] 3233-3240 (1992)

24. S Song, H M Jennings, Pore solution chemistry of alkali-activated ground granulated blast-furnace slag, Cem Concr Res, 29 (1999) 159-170.

Recibido: 04.12 .01

Aceptado: 30.01 .02 